AperTO - Archivio Istituzionale Open Access dell'Università di Torino

The relevance of "non-criteria" clinical manifestations of antiphospholipid syndrome: 14th International Congress on Antiphospholipid Antibodies Technical Task Force Report on Antiphospholipid Syndrome Clinical Features

This is the author's manuscript

Original Citation:

Availability:

This version is available http://hdl.handle.net/2318/1609748

since 2016-11-04T10:28:17Z

Published version:

DOI:10.1016/j.autrev.2015.01.002

Terms of use:

Open Access

Anyone can freely access the full text of works made available as "Open Access". Works made available under a Creative Commons license can be used according to the terms and conditions of said license. Use of all other works requires consent of the right holder (author or publisher) if not exempted from copyright protection by the applicable law. 
This Accepted Author Manuscript (AAM) is copyrighted and published by Elsevier. It is posted here by agreement between Elsevier and the University of Turin. Changes resulting from the publishing process - such as editing, corrections, structural formatting, and other quality control mechanisms - may not be reflected in this version of the text. The definitive version of the text was subsequently published in AUTOIMMUNITY REVIEWS, 14 (5), $2015,10.1016 / \mathrm{j}$.autrev.2015.01.002.

You may download, copy and otherwise use the AAM for non-commercial purposes provided that your license is limited by the following restrictions:

(1) You may use this AAM for non-commercial purposes only under the terms of the CC-BY-NC-ND license.

(2) The integrity of the work and identification of the author, copyright owner, and publisher must be preserved in any copy.

(3) You must attribute this AAM in the following format: Creative Commons BY-NC-ND license (http://creativecommons.org/licenses/by-nc-nd/4.0/deed.en), 10.1016/j.autrev.2015.01.002

The publisher's version is available at:

http://linkinghub.elsevier.com/retrieve/pii/S1568997215000038

When citing, please refer to the published version.

Link to this full text:

http://hdl.handle.net/ 


\title{
The relevance of "non-criteria" clinical manifestations of antiphospholipid syndrome: 14th International Congress on Antiphospholipid Antibodies Technical Task Force Report on Antiphospholipid Syndrome Clinical Features.
}

Abreu MM ${ }^{1}$, Danowski ${ }^{2}$, Wahl $\mathrm{DG}^{3}$, Amigo $\mathrm{MC}^{4}$, Tektonidou $\mathrm{M}^{5}$, Pacheco $\mathrm{MS}^{2}$, Fleming $\mathrm{N}^{6}$, Domingues $\mathrm{V}^{7}$, Sciascia $\mathrm{S}^{8}$, Lyra $\mathrm{JO}^{9}$, Petri $\mathrm{M}^{10}$, Khamashta $\mathrm{M}^{11}$, Levy $\mathrm{RA}^{12}$.

${ }^{1}$ Disciplina de Reumatologia, Universidade Federal do Rio de Janeiro, Rio de Janeiro, Brazil. Electronic address:

mirhelen.abreu@gmail.com.

${ }^{2}$ Serviço de Reumatologia, Hospital do Servidor Estadual do Rio de Janeiro, Rio de Janeiro, Brazil.

${ }^{3}$ Vascular Medicine Division and Regional Competence Centre For Rare Vascular and Systemic Autoimmune Diseases, Centre

Hospitalier Universitaire (CHU), Nancy, France; Institut Lorrain du Coeur et des Vaisseaux Louis Mathieu, Vandoeuvre-les-Nancy,

France; Institut National de la Santé et de la Recherche Médicale (INSERM), U1116, Vandoeuvre-les-Nancy Cedex, France.

${ }^{4} \mathrm{ABC}$ Medical Center, México City, Mexico.

${ }^{5}$ First Department of Internal Medicine, University of Athens, Athens, Greece.

${ }^{6}$ Pedro Ernesto University Hospital of the Universidade do Estado do Rio de Janeiro, Rio de Janeiro, Brazil.

${ }^{7}$ New York Presbyterian Hospital, Weill Cornell Medical College, New York, NY, USA.

${ }^{8}$ Centro di Ricerche di Immunopatologia e Documentazione su Malattie Rare and Università di Torino, Dipartimento di Scienze

Cliniche e Biologiche, Torino, Italy; Graham Hughes Lupus Research Laboratory, King's College London, London, UK.

${ }^{9}$ Rio de Janeiro Municipality, Rio de Janeiro, Brazil.

${ }^{10}$ Johns Hopkins University School of Medicine, Baltimore, USA.

${ }^{11}$ Lupus Research Unit at St Thomas' Hospital, London, UK.

${ }^{12}$ Discipline of Rheumatology, Universidade do Estado do Rio de Janeiro, Rio de Janeiro, Brazil.

\begin{abstract}
The purpose of this task force was to critically analyze nine non-criteria manifestations of APS to support their inclusion as APS classification criteria. The Task Force Members selected the non-criteria clinical manifestations according to their clinical relevance, that is, the patient-important outcome from clinician perspective. They included superficial vein thrombosis, thrombocytopenia, renal microangiopathy, heart valve disease, livedo reticularis, migraine, chorea, seizures and myelitis, which were reviewed by thisInternational Task Force collaboration, in addition to the seronegative APS (SN-APS). GRADE system was used to evaluate the quality of evidence of medical literature of each selected item. This critical appraisal exercise aimed to support the debate regarding theclinical picture of APS. We found that the overall GRADE analysis was very low for migraine and seizures, low for superficial venous thrombosis, thrombocytopenia, chorea, longitudinal myelitis and the so-called seronegative APS and moderate for APS nephropathy, heart valve lesions and livedo reticularis. The next step can be a critical redefinition of an APS gold standard, for instance derived from the APS ACTION registry that will include not only current APS patients but also those with antiphospholipid antibodies not meeting current classification criteria.
\end{abstract}

\section{Keywords}

Antiphospholipid Syndrome;

GRADE system;

Clinical features;

Non-criteria manifestations;

Accuracy 


\section{Introduction}

\subsection{Panorama of APS clinical criteria and diagnosis challenges}

Antiphospholipid syndrome (APS) classification process is characterized by the presence of antiphospholipid antibodies (aPL), namely anticardiolipin antibodies (aCL), anti-beta 2 glycoprotein I (anti-b2GPI), or lupus anticoagulant (LA) associated with thrombosis of arteries or veins or the microcirculation and/or well defined obstetrical manifestations. However, to date there are no accepted criteria for diagnosis of this condition, although classification criteria tend to be used for definition and for diagnostic purposes. Current classification criteria for definite APS were established in a workshop, preceding the 11th International Congress on aPL in Sydney. These criteria derived from the Sapporo preliminary classification criteria for APS [1].

Several clinical manifestations associated with aPL are not included in the revised classification criteria [1]. Since the Congress in Sydney, a significant body of basic research and clinical studies on APS has appeared, bringing new data and an opportunity to revise clinical as well as laboratory manifestations. Considering these new data, the main goal of this task force was to examine the body of evidence related to the set of clinical manifestations that are not included in the current criteria and their association with aPL.

In this context, the Antiphospholipid Antibodies Task Force on Clinical Manifestations was carried out from January to September, 2013 to examine data, the quality of evidence according to GRADE system and develop recommendations in order to carefully suggest a revision of the current classification criteria [2]. A workshop held in Rio de Janeiro at the 14th International Antiphospholipid Congress discussed the findings [3] and [4]. We examined all potential clinical criteria and selected the most relevant ones, we did not discuss the obstetrical manifestations, the future trends in treatment and the catastrophic APS which were evaluated by other groups [5], [6] and [7].

\section{Objectives}

The objectives of this task force were: (1) to assess the patient-important outcomes that have been considered as non-criteria APS manifestations, and (2) to assess the body of evidence of each outcome to support the recommendation to be included on APS classification criteria.

\section{Methodology}

The PICO question was formulated as: "In patients with APS $(P)$, are non-criteria APS manifestations $(I)$ associated with APS classification, leading to earlier and most adequate treatment intervention to improve the patient-important outcomes $(O)$ "'?

We aimed to define and assess the outcomes that may be related to each non-criteria manifestation. An international group of APS experts of different specialties suggested non-criteria APS manifestations that were considered relevant. Some of the authors presented the current evidence in their area of expertise. Finally, during the Meeting, there was a debate in order to evaluate the proposal to include each manifestation as part of updated APS classification criteria. 
The outcomes were selected from the disease perspective. The importance of the outcome was evaluated according to its consequence to clinical decision-making (patient-important outcome). We considered the following: (1) the occurrence of this outcome on the disease in question and (2) the influence that it has to change the clinical care plan [8].

The categories were: critical for decision making (when it indicates risk of mortality), very important, but not critical for decision-making (when it indicates risk of disabilities, but no risk of death) and low importance for decision making (when it indicates transitory and slight discomfort to the patient). We have elaborated one PICO for each outcome.

We followed three steps in considering the relative importance of outcomes: (1) preliminary classification of outcomes, before reviewing the evidence; (2) reassessment of the relative importance of outcomes after reviewing the evidence; and (3) judgment of the balance between the desirable and undesirable intervention effects.

After the outcomes were chosen and discussed, the quality of the body of evidence was evaluated and summarized. The quality evaluation was based on the study design and the presence of methodology limitations. The dimensions that covered the assessment of the limitations were risk of bias; indirectness of evidence; inconsistency of results; imprecision of results and publication bias.

It is important to emphasize the 'directness' dimension and how it was assessed in this task force. Directness is defined by whether the populations included in the studies correspond to the population in focus for the decisions under debate or not. Such an evaluation is difficult and could not be assessed objectively by this task force. To limit this bias, we first defined the PICO of each manifestation. Then, we defined the outcome(s) measured for each manifestation and it's (their) relative importance for the patient, from a clinician's perspective. This categorization was based on the discussion developed during the meeting with all participants, combined with the quality of evidence (GRADE system) to support the assumption that they could be associated with APS classification. The next step was the analysis of the overall quality of evidence for the manifestations selected and their correlated outcomes. This step provided a consensus approach of the current knowledge regarding each manifestation, the gaps of evidence and the framework to provide the recommendations and further steps on future studies. The noncriteria manifestations included in the debate were: superficial vein thrombosis, thrombocytopenia, nephropathy, heart valve disease, migraine, chorea, transverse myelitis and seizures, and we also discussed the so-called seronegative APS (SN-APS). The results below are related to the assessment of the quality of body of evidence for the outcomes linked to each non-criteria manifestation to map the current knowledge about them, including their gaps of the evidence. It will allow us to construct the assumptions for further studies of APS classification criteria revision. Two independent investigators have performed data abstraction and quality assessment, and compared their results. The quality of evidence obtained was assessed using the GRADE approach. 


\section{Results}

\subsection{Superficial vein thrombosis}

PICO specific question: "In patients with APS, is superficial vein thrombosis associated with recurrent events, and would this criterion lead to earlier and most adequate treatment intervention to prevent the occurrence of recurrent thrombotic events, including serious life threatening events"?

PICO: P - APS; I/C — superficial vein thrombosis manifestations; $\mathrm{O}$ - the occurrence of recurrent thrombotic events, including serious life threatening event.

\subsubsection{Patient-important outcome}

Superficial vein thrombosis (SVT) is a common disease, characterized by a thrombotic process in a superficial vein. The incidence is higher than of deep-vein thrombosis (DVT) (1/1000). SVT shares many risk factors with DVT, but affects twice as many females and frequently occurs in varicose veins. About $8 \%$ of the patients with SVT experience symptomatic thromboembolic complications within three months, still most individuals with isolated SVT (without concomitant DVT) are commonly treated with low dose anticoagulation for a short term. Risk factors for concomitant DVT are recent hospitalization, immobilization, autoimmune disorders, age older than 75 years, prior DVT, cancer and SVT in nonvaricose veins. Risk factors for the occurrence of complications are male gender, history of venous thrombo-embolism (VTE), cancer, SVT in non-varicose vein or involving the sapheno-femoral junction. Ultrasound assessment and exclusion of DVT is essential [9].

Considering all of these aspects and the scarcity of evidence regarding SVT and aPL/APS correlation, the 'occurrence of serious thrombotic consequences from SVT' was defined as an important but not critical outcome. This categorization was corroborated by its exclusion in the revised classification criteria. Due to the limited data available at that time, but also to avoid over-classification of other diseases as APS in particular Behçet's disease in which SVT and aPL may coexist.

\subsubsection{Findings}

There were three studies included: two cohort studies in aPL/APS patients and one clinical trial in the general population. The first cohort study investigated clinical and serological features in patients with APS. They found that SVT occurred in 9.1\% of the patients with APS [10]. The second cohort explored the risk factors for thrombotic events after 35 months of follow-up in patients with aPL. Although seemingly benign, SVT predicted a 7.5-fold increased risk for incident thrombotic events in aPL patients. The univariate analysis showed that patients with both aPL and activated protein C resistance have an increased risk for thromboembolic events (hazard ratio [HR] 3.67 [95\% confidence interval [CI] 1.3110.31]). The multivariate analysis found that SVT remained an independent risk factor (HR 7.45 [95\% CI 2.25-24.66]) for further thromboembolic events.

As evidence supporting treatment of isolated SVT was sparse and of poor quality, the large, randomized, double-blind, placebo controlled (RCT) CALISTO trial was conducted in the general population assessing the effect of fondaparinux on symptomatic outcomes in isolated SVT [11]. This study showed that, compared to placebo, $2.5 \mathrm{mg}$ fondaparinux given for 45 days reduced the risk of symptomatic 
thromboembolic complications by $85 \%$ without increasing bleeding. Based on CALISTO and other observational studies, evidence-based recommendations can be made for most SVT patients. Further studies can now be performed in higher risk patients including patients with aPL to address unresolved issues.

\subsubsection{Overall quality of evidence and recommendations}

The overall quality of evidence was GRADE high, at the start with two cohort studies and one RCT in the general population. However there were limitations, as the patients included in the RCT were not directly representative (general population), decreasing it to GRADE moderate. Additionally, there were few studies, making the recommendation support vulnerable, with risk of interpretation bias. Based on these studies, there is low quality of evidence that suggests that SVT can be included in APS classification.

\subsection{Thrombocytopenia}

Specific question: "In patients with APS $(P)$, is thrombocytopenia $(I)$ associated with APS classification, leading to earlier and most adequate treatment intervention to prevent the occurrence of recurrent thrombotic events and other complications including serious life threatening event $(O)$ "'?

PICO: P - APS; I/C - thrombocytopenia; $\mathrm{O}$ - the occurrence of recurrent thrombotic events, and other complications including serious life threatening event.

Patient-important outcome: occurrence of recurrent thrombotic events and other complications.

Preliminary relative importance: critical for decision-making.

\subsubsection{Patient-important outcome}

Clinicians and researchers have shown that thrombocytopenia is frequent in APS (20 to 46\%) but its paradoxical association with thrombosis is noteworthy. This information is relevant to practical decisions such as prevention of recurrent thrombosis and may guide treatment decisions. Additionally, the assumption that patients with thrombocytopenia and aPL are at high risk for developing APS contributes to considering this outcome (recurrent thrombotic events) as critical for decision making.

\subsubsection{Findings}

Eleven cohort studies were included. They investigated, respectively: the frequency of thrombocytopenia in APS patients; the frequency of aPL positivity in patients with thrombocytopenia; and the clinical importance of idiopathic thrombocytopenic purpura (ITP), and how positive aPL in patients with thrombocytopenia is clinically important for decision making. The patient-important outcome was the occurrence of recurrent thrombotic events and it was categorized as critical for decision-making.

Several studies demonstrated a high prevalence of aPL in ITP patients and some explored its possible correlation with thrombosis [12]. Yang et al. performed an observational prospective study that aimed to determine the frequency and clinical implications of elevated aPL in adult patients with ITP. The authors identified aPL positivity in $28.5 \%$ of the patients in this ITP cohort [13]. 
Observing that some patients with ITP paradoxically had an increased risk of thrombosis when therapeutic interventions corrected thrombocytopenia Kim et al. conducted a retrospective study that investigated the prevalence and clinical significance of aPL in ITP patients and assessed the risk factors for thrombosis. They found aPL in $41.6 \%$ of 165 patients with ITP; patients with aPL had an increased incidence of thrombosis $(21.7 \%$ vs. $6.2 \%)$, LA and hypertension were independent predictors of thrombosis [14]. Danasu et al. conducted a retrospective analysis that explored aPL positivity in patients with isolated thrombocytopenia and correlated this finding with the occurrence of thromboembolic (TE) events. Thrombosis was observed in $22 \%$ of 64 patients evaluated, $14 \%$ with aPL [15]. Diz-Küçükkaya et al. conducted a prospective cohort study where the prevalence and clinical significance of aPL were investigated in 82 patients with ITP. Eighty-two newly diagnosed ITP patients were prospectively studied. They were evaluated for the presence of LA and aCL IgG and M. Thirty-one patients (37.8\%) had a positive aPL at diagnosis. After five years of follow-up, cumulative thrombosis-free survival of aPL-positive $(\mathrm{n}=31)$ and aPL-negative $(\mathrm{n}=51)$ ITP patients was $39 \%$ and $97.7 \%$, respectively. A significant difference was found between these groups by log-rank test $(p=0.0004)$. LA was an important risk marker for the development of thrombosis in ITP patients. After an average follow-up of 38 months, 14 ITP patients (45\%) who had aPL positive developed clinical features (thrombosis or fetal losses) related to APS. There were no differences between the aPL-positive patients with and without APS regarding the initial platelet counts, response to therapy, or aCL positivity. LA was significantly more frequent in those with ITP who developed APS ( $p=0.0036$; relative risk [RR] 7.15; 95\% CI 1.7-47) [16]. The exact frequency and clinical meaning of aPL in ITP patients is still unknown. A single center retrospective cohort assayed aCL, LA and anti-b2GP1 in 215 recently diagnosed ITP patients. Analyzing the risk factors for thrombosis, the multivariate analysis showed that thrombotic events were associated with age (HR 1.6; 95\% CI 1.2-2.4), LA (HR 9.9; 95\% CI 2.3-43.4) and high level IgG-aCL level (HR $7.5 ; 95 \%$ CI 1.8-31.5). The meaning of this result is that all the patients with aPL who developed a thrombosis were less than 45 years of age whereas nine of the 10 patients without aPL who did have a thrombotic event were over 45 years of age $(p=0.005)$ [17]. In contrast, in another ITP study, aPL were found in $46.3 \%$ and after a relatively short follow-up period, individuals with elevated aPL neither showed nor had a history of thrombosis. Because of this finding, the latter authors do not support a role for aPL in the pathogenesis of ITP [18].

Since thrombocytopenia is an important feature of systemic lupus erythematosus (SLE), it is crucial to determine if it is also associated with primary APS (PAPS). The first report from the Italian aPL registry described that the prevalence of thrombocytopenia in patients with PAPS was similar to that seen in patients with SLE associated APS [19]. In 1997, Cuadrado et al. analyzed retrospectively the prevalence, clinical and serological associations of thrombocytopenia in 171 APS patients. Severe thrombocytopenia $(<50 \times 10 \%$ L) was detected in six (17.6\%) patients at some point during the follow-up period. Also, a higher percentage of SLE associated APS (55\%) was found in the thrombocytopenic group. Both results were not statistically significant, and maybe limited by the small sample size [20]. Thrombocytopenia in PAPS is still a gray zone of evidence. In the Euro-Phospholipid Project, thrombocytopenia was found in $21 \%$ of PAPS patients. This project started in 1999 as a multicenter, consecutive and prospective designed trial. A total cohort of 1000 patients with APS, derived from 13 
countries, has been followed since then. Although thrombocytopenia was correlated to patients with APS associated with SLE, it was not correlated in patients with primary APS [16]. A prospective Serbian cohort study showed that thrombocytopenia was observed significantly more frequently in patients with secondary APS, when compared with primary APS group, which corroborates the findings from other studies [21].

Comellas-Kirkerup et al. retrospectively reviewed 187 consecutive, patients with primary APS from a tertiary care center. This registry included APS patients as well as patients with positive aPL and noncriteria clinical manifestations. Fifty-five patients (44 women, 80\%) had hematological manifestations. Thirty-five patients (64\%) had thrombocytopenia, 14 (25\%) had autoimmune hemolytic anemia and 6 $(11 \%)$ had Evan's syndrome. The authors analyzed the frequencies of LA in patients with or without definite APS with hematologic manifestation. They found that patients with thrombocytopenia, including two with Evan's syndrome, from the clinical APS group were 2-fold more frequently positive for LA (71\%) than from the non-clinical APS group (35\%; OR 4.46; 95\% CI 1.11-17.90; $p \leq 0.05)$ [22]. Three hundred and seven APS patients were retrospectively evaluated by Krause et al., most patients had PAPS ( $\mathrm{n}=173,56.1 \%)$ and in 104 patients APS was associated to SLE (33.9\%); 90 patients had thrombocytopenia (29.3\%). Thrombocytopenia was significantly more frequent in APS patients with SLE when compared to PAPS patients $(41.9 \%$ vs. $23.1 \%, p=0.001)$. Significant associations were found between thrombocytopenia and cardiac valves thickening and dysfunction, epilepsy, chorea, arthritis, livedo reticularis and skin ulcerations. In contrast, the rates of thrombotic episodes as well as obstetric complications were similar in patients with and without thrombocytopenia. These data suggest that thrombocytopenia may be a risk factor for cardiac, neurological and cutaneous manifestations in APS patients [23]. Currently, an ongoing International Clinical Database of aPL-positive patients (APS ACTION) is enrolling at least 1000 patients to be followed over 10 years. Non-criteria aPL related manifestations such as thrombocytopenia will be investigated [24] and [25].

\subsubsection{Overall quality of evidence and recommendations}

For the clinical practice, considering the body of evidence, it starts as GRADE moderate as one study had patients with other diseases included. Relevant cohorts were included, most of all with large follow-up, substantial sample size (in view of the low prevalence of the disease), with an elegant methodology (defined by the checklist of the critical appraisal methodology) and robust magnitude of effect, translated by the statistically significant and clinically meaningful findings. One study included a small sample size of several subgroups whose results were imprecise, and another study with short follow-up and with no correlation to any important outcomes. The final evaluation was GRADE low. The evidences have shown that thrombocytopenia does not reduce the risk of further thrombosis.

These evidence support a suggestion that thrombocytopenia should be considered as a classification criterion of APS. Further research shall be conducted in order to better understand these findings and therapeutic consequences. 


\subsection{Renal microangiopathy}

Specific question: "In patients with APS $(P)$, is APS Nephropathy (APSN), defined by thrombotic microangiopathy (TMA), associated with APS classification (I), leading to earlier and most adequate treatment intervention to prevent the occurrence of kidney failure or death from kidney failure $(O)$ "? PICO: P — APS; I/C — APS Nephropathy (APSN), defined by thrombotic microangiopathy (TMA); O — kidney failure or death from kidney failure.

Patient-important outcome: kidney failure or death from kidney failure.

Preliminary relative importance: critical for decision-making.

\subsubsection{Patient-important outcome}

The kidney is currently recognized as a major target organ in both PAPS and SLE-related APS. The outcome of interest here is kidney failure or death due to kidney failure. APS nephropathy (APSN), defined by thrombotic microangiopathy (TMA), characterized by fibrin thrombi in glomeruli and/or arterioles, has been the most frequently reported intra-renal vascular lesion in aPL-positive patients with or without lupus. Additionally, from a clinical perspective, renal manifestations have in common between SLE and APS the high prevalence of systemic hypertension and proteinuria. Although these diseases may have other clinical presentations, they can be superimposed. From a clinical practice point of view, the clear definition of whether such a renal presentation is related to SLE or APS will determine distinct treatment decisions. Taking this into account, kidney failure and/or death by kidney failure was defined as very critical outcomes.

For the assessment of the dimensions that determine the quality of evidence, we might question in the first place how closely APSN is correlated with the patient-important outcome that would lead to decision changes in order to improve it. If the evidence points toward a close relationship between APSN and patient-important outcomes (here, kidney failure and/or death by kidney failure), then the quality of evidence should not decrease. TMA seems to be the surrogate marker of interest for the patient-important outcome that precedes APSN and should be used as the surrogate and the relationship between TMA and kidney failure - the outcome we want to address. So, the question is if either APSN or perhaps TMA is correlated with patient important outcomes and how likely it is that it progresses to disease [26]. This reasoning guided the judgment of the quality of the body of evidence.

\subsubsection{Findings}

To quantitatively support this judgment, we looked at the findings from the previous task force [27]: among primary APS patients with renal involvement, APSN was observed in $100 \%$ of cases in almost all considered studies. Summary data from all the studies that examined patients with positive aPL (with or without APS) in comparison with those without aPL showed a higher frequency of APS nephropathy in those patients with circulating aPL $(p<0.001)$. 
Most renal lesions in APS have been described as intra renal thromboses, but there remains a lot to be learned concerning the spectrum of APSN: the frequency, severity, symptomatology, and even the basic histology of these lesions.

To support the assumption that TMA can be the surrogate of interest for the patient-important outcome that precedes APSN, animal/in vitro studies were included in this analysis. Nangaku et al. presented consistent results that confirm earlier studies, which establish a significant protective role for CD59 in modulating immune glomerular injury in vivo. They extended their observations by localizing the effect to the GEN in the model they described of immune TMA induced by the renal artery perfusion of antiglomerular endothelial cell (anti-GEN) antibody. These findings support the hypothesis that modulation of CD59 activity may have important benefits in multiple diseases in which anti-endothelial antibodies may be pathogenic [28]. Using different mouse monoclonal and human aPL antibodies, Seshan et al. developed an original animal model of renal injury that shares many features with TMA. They described more than one mechanism/signaling pathway involved in glomerular injury induced by aPL antibodies. Both complement-dependent and -independent pathways were identified to induce glomerular endothelial cell damage and renal function impairment [29]. While theories can be constructed around any possible mechanism, a theory is only as good and informative as the data that support it (and from which it may be derived). Considering this, these findings together build the hypothesis for the valid clinical diagnostic studies that may provide the contextual link of whether or not these theories were confirmed.

Several studies have been corroborating the assumption that TMA is a surrogate for APSN and, consequently, for kidney failure by APSN. In a recent long follow-up study examining the significance of the inclusion of renal vascular lesions in the ISN/RPS classification system for lupus nephritis, TMA was associated with the poorest outcome regarding the five and ten year renal survival [30]. The findings presented by this study add to the current knowledge the notion that the renal lesions in the series are representative of those seen in PAPS and are correlated to high titer aCL. TMA can be a relevant outcome to be measured in future studies from different perspectives.

For this review, six papers were included; the in vitro studies presented above were not included on GRADE analysis, but they corroborate this construct. The main questions were which clinical and histopathological lesions can be found in renal biopsy that define a morphologic picture suggestive of APS and its implication on clinical decision-making.

Amigo et al. found renal disease in $25 \%$ of the 20 patients with PAPS evaluated at a cross-sectional study whose dimension of directness for the question addressed in this outcome was contemplated. It is justified, as only APS patients were included, carefully excluding SLE from the sample. TMA — the surrogate marker of the outcome evaluated here - was investigated in those with definite APS classification with renal clinical findings (proteinuria, hypertension and renal failure). There are some limitations of this pioneer study that reduces the quality of evidence on the context of the body of evidence for this task force. On the other hand, biopsy findings were consistent with a TMA involving both arterioles and glomerular capillaries. These demonstrations would reduce the presence of some plausible residual confounding, which would suggest a spurious effect if no effect was observed [31]. 
In 1999, Nochy et al. retrospectively examined the kidney biopsies of 16 patients with PAPS in a multicenter cohort study and followed-up for five years or more. This group explored the clinical and histopathologic lesions on renal biopsies, which define a morphologic picture that should suggest the diagnosis of APS. All 16 patients had positive tests for LA, aCL or both. In all cases of PAPS, there was a vascular nephropathy characterized by small vessel vaso-occlusive lesions associated with fibrous intimal hyperplasia of interlobular arteries (12 patients), recanalizing thrombi in arteries and arterioles (six patients), and focal cortical atrophy (ten patients). They also described extra renal manifestations related to thrombosis and that they were likely to be correlated to high aCL levels ( $62 \%$ of patients $>40 \mathrm{GPL}$ units), linked to the severity of the thrombotic process. Moreover, the extra-renal clinical involvement in these patients corresponded to that described in PAPS. Although this small sample may provide inconsistent and imprecise results, the ensemble of histologic renal lesions defined in this study should aid in the separation of the lesions found in cases of secondary APS, especially SLE, into those lesions related to APS. This information contributes to support decisions on clinical practice, despite these limitations [32].

Defining the nature of renal disease in lupus in the context of APS represents a challenge to practitioners and Daugas et al. investigated whether the renal histopathologic lesions of APS could be as accurate as NSLE to discriminate the nature of the nephropathy in patients with SLE-APS. To address this question, they performed a second retrospective cohort study to determine: (1) if APSN as the group have described on the previous study could be found in the most frequent secondary form of APS, associated with SLE; (2) if some patients with SLE have risk factors associated to the development of an APSN; and (3) if APSN is a superadded renal morbidity factor in lupus patients. They included 114 patients that divided in seven groups of patients according to clinical, obstetrical and laboratory criteria. They found that APSN in SLE is independent of the class of lupus glomerulopathy. Thirty-six patients (32\%) presented one or more histologic lesions of APSN: 20 patients (18\%) had acute lesions of APSN, of whom nine patients had acute APSN alone, four patients with acute APSN and fibrous occlusion and ischemic cortical atrophy (FCA); 27 patients (24\%) had chronic lesions of APSN, of whom 17 (15\%) had associated FCA; eleven patients had both acute and chronic APSN. In none of the patients in whom it was identified FCA as the exclusive lesion suggestive of APSN. For accuracy studies, wide confidence intervals for estimates of test accuracy or true and false positive and negative rates can reduce quality of evidence (imprecise evidence dimension). In this study, statistical tests revealed significant associations between the presence of APS or a positive test for LA and the existence of an APSN (OR 6 and 5.9, respectively; $p=0.0001$ for both), a chronic APSN (OR 3.8, $p=0.005$ and OR $=5.7, p=0.0001$, respectively), an acute APSN (OR $3.7, p=0.026$ and OR 3.8, $p=0.007$, respectively), or FCA lesions (OR 9.2, $p=0.0002$ and OR $8.2, p=0.0001$, respectively). However, in contrast with LA, aCL showed no significant association with APSN, chronic APSN, acute APSN, or FCA lesions, no matter the aCL titer (median, 28 GPL units [0760] for patients with APSN compared with 23 GPL units [0-178] for patients without APSN [ $p=0.77]$ ). Only three patients (15\%) with neither aCL nor LA (non-aPL group) showed APSN, giving a negative predictive value of $85 \%$ for aCL and LA in predicting APSN. In conclusion, these data illustrate that limitations due to imprecision and inconsistencies were absent [33]. We could not find limitations in this study and it is important to emphasize that its relevance is not only because it established the existence of 
APSN within SLE, but it has also shown that these lesions belong to the group of APS-related vascular manifestations independent of SLE. Its presence in conjunction with lupus nephropathy probably increases the risk of evolution toward end-stage renal disease. This information may contribute to define clinical practice decisions.

Tektonidou et al. explored a further understanding about the clinical and laboratory associations in patients with SLE associated with APSN [34]. Additionally, this team examined the frequency of APSN in patients with SLE. APS nephropathy was diagnosed in 32 (39.5\%) of 81 SLE patients with aPL, compared with only three $(4.3 \%)$ of the 70 SLE patients without aPL $(p=0.01)$. The prevalence of APSN in SLE patients with secondary APS and in SLE/non-APS patients with aPL was $67 \%$ and $31.7 \%$, respectively. A strong association between APS nephropathy and APS, LA [OR 11.46 (CI 95\% 3.141.7)], aCL [OR 5.7 (CI 1.8-17.8)], and hypertension [OR 4.0 (CI 1.5-8.6)] was noted. Odds ratio (OR) calculations were based on the risks of APSN, given the absence or presence of the clinical and laboratory findings. These findings also corroborate the initial studies by Glueck [35] and Kant [36], which found that TMA in renal biopsies of SLE patients was associated with LA and thrombotic events.

Later, the same group (2008) examined the renal histological, clinical and laboratory characteristics of different groups of patients with APS including catastrophic APS (CAPS)[37]. The study included CAPS $(\mathrm{n}=6)$, PAPS $(\mathrm{n}=8)$, and SLE-APS $(\mathrm{n}=23)$ patients with biopsy-proven renal involvement. Regarding PAPS and SLE-related APS patients, TMA was detected in $37.5 \%$ and $35 \%$ of patients, respectively, while the frequencies of FIH and FCA were similar to those observed in CAPS patients $(p>0.99)$. This was the first time that the renal histologic, clinical, and laboratory characteristics of three different groups of APS patients with biopsy-proven renal involvement was compared the pertinent data. The most frequent findings in all three APS groups were hypertension, proteinuria, hematuria, and renal insufficiency. The presence of the same characteristics of APSN among all three groups of APS patients suggests an association between this nephropathy and APS. According to these authors, APSN should be included in the classification criteria for definite APS, and the use of an appropriate treatment in these patients should be examined. However, for a better understanding of the TMA role in patients with SLE, the renal SLE outlook was assessed by Song et al. They explored the clinical, laboratory and pathological features as well as risk factors for clinical outcomes of patients with TMA in lupus nephritis [38]. They presented a detailed histopathological analysis of a series of 148 patients with biopsy-proven lupus nephritis, focusing on the prevalence of TMA and found such lesions in 36 out of the 148 patients with lupus nephritis. Although it is indirect, which decreases the overall quality of evidence, this study corroborates the knowledge that patients with SLE can develop other kidney disorders that may be related or not to SLE.

\subsubsection{Overall quality of evidence and recommendations}

The critical appraisal of these studies shows that their methodologies have several strengths that draw our attention. First, the studies' designs are appropriate for valid diagnostic studies. On average, patients have clear recruitment criteria and address clear-cut PAPS. They all meet the classification criteria for PAPS; some studies included APS versus SLE comparison. Additionally, one study that is focused on SLE was included on this analysis as it corroborates the body of evidence. 
When it comes to assessment limitation, the judgment regarding the presence of indirectness in this last study represented the greatest challenge for this workgroup. APSN is closely correlated with kidney failure - the patient-important outcome in focus. TMA is assumed as a surrogate of interest for the patient important outcome that precedes APSN. Also, aPL profile is assumed as a surrogate of the disease in question, that is APS. Overall GRADE started as high and decreased to moderate because of this. As it is a very critical important outcome whose definition will imply in intervention that will change the outcome, we assumed that this indirectness of sample selection (SLE) addresses the questions for this patient-important outcome; it decreases even more the overall quality of evidence.

Therefore, there are bodies of evidence with moderate quality to support a recommendation that APSN should be included in the APS classification. Additionally, gaps of knowledge were identified suggesting that future research directions need to include prospective multicenter studies that analyze APSN histological criteria. Large-scale studies will be relevant for the identification of the characteristics of APSN associated with worse renal prognosis. The effect of anticoagulation or targeted treatments (to C5a, for example) on long-term outcome of APSN should also be examined in prospective randomized studies.

\subsection{Heart valve disease}

Specific question: "In patients with APS, are heart valve lesions defined by (a) valve thickness $>3 \mathrm{~mm}$; (b) localized thickening involving the proximal or middle portion of the leaflets; or (c) irregular nodules on the atrial face of the mitral valve and/or the vascular face of the aortic valve associated with APS classification (I), leading to earlier and most adequate treatment intervention to prevent the occurrence of heart failure, valve replacement or death from heart failure/valve replacement"?

PICO: P — APS; I/C — valve lesions defined by (a) valve thickness $>3 \mathrm{~mm}$; (b) localized thickening involving the proximal or middle portion of the leaflets; or $(c)$ irregular nodules on the atrial face of the mitral valve and/or the vascular face of the aortic valve; $\mathrm{O}$ - heart failure, valve replacement or death from heart failure/valve replacement.

\subsubsection{Patient-important outcome}

According to the editorial published by Petri M, using these strict requirements for evidence-based criteria, cardiac valvular disease (thickening or vegetations) should clearly be included in APS criteria. Multiple supportive prospective cohort studies and case-control studies, were presented which corroborate the existence of a strong pathophysiologic rationale for considering cardiac valve disease as a manifestation of APS [39]. Positive associations have been found using both study designs and in both SLE and non-SLE populations, meeting the criteria for inclusion in evidence-based classification criteria. With the emphasis, however, that it is necessary for valvular disease to be stringently defined as valve thickening or valve vegetations [40].

In the context of APS, valve lesions are defined by (a) valve thickness $>3 \mathrm{~mm}$; (b) localized thickening involving the proximal or middle portion of the leaflets; or (c) irregular nodules on the atrial face of the mitral valve and/or the vascular face of the aortic valve. Its consequence in clinical practice is heart failure, valve replacement or death from heart failure. 
From the disease perspective, it is crucial to identify whether heart valve disease is present or not, as it will imply aggressive treatment care plan [41]. The patient-important outcome from the clinical perspective is heart failure, heart valve replacement or death from heart failure/valve replacement and these outcomes are categorized as very critical for decision-making. To illustrate this importance on clinical practice, a case report of a 49 year-old woman with a history of rheumatic fever at 14 years of age described by Espinola-Zavaleta is presented here. At 49, a yearly routine two-dimensional transthoracic and transesophageal examinations and three-dimensional reconstruction were performed, which made it possible to dismiss a rheumatic etiology for the valve lesions, as large nodules were found on the free edges of both mitral leaflets without thickening or fusion of the subvalvular apparatus. Because of the echocardiographic evidence of probable PAPS, an immunological profile was performed. Findings included negative rheumatoid factor, negative antinuclear antibodies and negative aCL with strong positive anti-b2GP1 and false positive VDRL [42]. This case is interesting as it involves a woman who suffered her first episode of rheumatic fever at the age of 14 years. It was the echocardiogram that first suggested an autoimmune disease and specifically APS because nodules were found on the free borders of both mitral leaflets and aortic valve leaflets without evidence of thickening or fusion of the subvalvular apparatus. The diagnosis of PAPS was confirmed by identifying antibodies against b2GP1 in the clinical evidence of SLE. This case report calls for attention for the consequences of misdiagnosis for the patients and for the potential role of echocardiographic and immunologic tests in establishing the diagnosis of APS with valve lesion and making it possible to distinguish it from rheumatic heart disease.

\subsubsection{Findings}

Ten studies were included in this analysis, of which two are case-control studies, two cross-sectionals, three cohorts, one randomized clinical trial and two systematic reviews. The main research questions regarded the clinical and serological features in long-term of cardiac manifestations in patients with APS; the frequency; how to identify it with accuracy, in order to discriminate APS-related heart valve disease of rheumatic and infectious heart valve disease; which clinical associations could be expected and the risk factors that were involved. The outcome measurements were, respectively, clinical questionnaires; echocardiography; and SPECT study (myocardial perfusion) Additionally, there were studies that analyzed antinuclear antibodies, serum levels of aCL and anti-b2GP1 antibodies (IgG and IgM) measurements for correlations analysis.

A case-control study published in 1996 described the prevalence and clinical significance of aPL in heart valve disease in order to determine whether the presence of aPL antibodies carries an increased risk for thrombotic events [43]. Eighty-nine consecutive patients and 80-matched control subjects were tested for $\mathrm{IgG}$ and $\mathrm{IgM} \mathrm{aCL}$ and for LA. The prevalence of aPL antibodies was significantly higher in patients (19 [21\%] of 89 ) than in controls ( $79 \%]$ of $80 ; p<0.05)$. Patients were divided into two subgroups according to the presence (A) or the absence (B) of aPL antibodies. No significant difference in age or gender ratio was observed between the two subgroups. A history of arterial thrombosis was more frequent in Group A (8 [42\%] of 19) than in B (8 [11\%] of 70) $(p<0.01)$. No significant difference with respect to the occurrence of thrombotic events was observed during an average follow-up period of 8.7 months. 
Thus, a high prevalence of aPL antibodies was found in patients referred for heart valve replacement compared with matched controls. No increased risk has been demonstrated in the patients with aPL antibodies.

The evolution of valve involvement and myocardial dysfunction over time in patients with SLE with or without aPL and/or APS was described on a 10-year cohort that included 17 patients with PAPS, 23 with SLE-associated APS (SLE/APS), 19 with SLE positive for aPL without APS, and 23 with SLE negative for aPL who were re-evaluated in the present echocardiography study [44]. Valvulopathy was measured by transthoracic echocardiography and it was detected in $65 \%$ of PAPS and $62 \%$ of SLE patients with or without aPL. Disease duration (OR 1.63; 95\% CI 1.13-2.36; $p=0.009$ for every five years of increase) and presence of SLE/APS (OR 3.51; 95\% CI 1.27-9.67; $p=0.015$ ) were the only factors associated with the progression of valvular disease by univariate and multivariate analyses. The consequence of valvulopathy to the patient (the importance of this outcome for the patient) was examined, showing that left ventricular diastolic dysfunction similarly progressed over time, with deceleration time (DT) and isovolumetric relaxation time (IVRT) being equally prolonged in the four groups $(p<0.05)$. Right ventricular DT was significantly prolonged in all three SLE patient groups $(p<0.001)$, whereas IVRT increased only in SLE/APS patients $(p=0.040)$.

The Serbian National Cohort study started in 2000 and analyzed cardiac manifestations in 374 patients (260 PAPS patients and 114 SLE/APS) during 10 years of follow-up [45]. The outcomes measured were aPL profile and cardiac manifestations, that were examined by echocardiography features. They found $30.7 \%$ secondary APS patients and 9.2\% PAPS patients with pseudo-infective endocarditis ( $p=0.0001$ ). Cardiac manifestations were observed in $28.7 \%$ of the patients who had more than one type of antibody, although it was not statistically significant $(p=0.78)$. Age was a significant factor for cardiac manifestations in APS ( $p=0.001)$ and, finally, aCL IgG and IgM positivity was related to valvular changes in all APS patients and high aCL levels increased the risk of these manifestations.

Clinical associations are also relevant for this topic. Krause that examined the association between cardiac and central nervous system, exploring cardiac valve disease; migraine (recurrent severe unilateral and bilateral headache); and epilepsy/seizures (simple partial, complex partial, or generalized tonic-clonic) using Doppler color flow echocardiographic and neurologic examinations [46]. The cardiac valve abnormalities in APS patients with cerebral vascular accident (CVA) were (for 284 sample): valve vegetation $(p<0.04)$; valvar thickening and dysfunction $(p<0.001)$ and for PAPS $(\mathrm{n}=159)$ the abnormalities found were: valvar thickening and dysfunction $(p<0.03)$; and overall cardiac valve abnormalities $(p<0.02)$. A significant association was also found between all valvar lesions and stroke or transient ischemic attacks (TIA), although they found a borderline association between valvar vegetations and migraine $(p=0.09)$.

Two 5-year transesophageal echocardiographic studies in primary APS demonstrated the progression of valve lesions and the ineffectiveness of oral anticoagulants and aspirin in terms of valvular lesion regression [47]. One of these prospective studies also investigated risk factors for the worsening or appearance of new valvular lesions over this 5-year follow-up. This study enrolled 56 APS patients. Before recruitment, 27 (48\%) had had one or more episodes of arterial or venous thrombosis, 29 (52\%) recurrent thromboembolic events, and eleven (20\%) had had just one fetal loss. Follow-up: three (5\%) 
died; all had high aCL titers (> 40 GPL). High IgG aCL titers: 30 patients (54\%) and high IgM aCL titers in $21(38 \%)$. LA was detected in 47 patients (84\%), and thrombocytopenia in 18 (32\%). At baseline evaluation, TEE showed $64 \%$ of cardiac involvement (valvular thickening or vegetations and/or potential embolic sources) in 34 patients. Over the 5-year follow-up cardiac involvement was unchanged in 30 subjects (64\%). New cardiac abnormalities: 17 patients (36\%), $15(88 \%)$ with high IgG aCL and two (12\%) with low IgG aCL titers. To corroborate the consistent and precise results, a multivariate logistic regression was carried out, evidencing that high IgG aCL represents the only independent risk factor for new and progressive cardiac abnormalities in APS patients [OR 4.5, 95\% CI 1.70-15.05].

The accuracy of the measurement tool is also a research theme on the context of valve manifestation in APS, as it might have to discriminate to other conditions with implications for practical decision-making, as in the context of cardiac valve manifestation in SLE patients. Using a spectral Doppler, Amoroso et al. examined the prevalence of cardiac alterations detectable by transthoracic echocardiography (TTE) and the possible role of aPLs in determining heart damage [48]. The outcome in this study was valvular impairment, defined only when it was hemodynamically relevant. Thirty-four SLE patients and 34 controls were analyzed. They observed that 19 (56\%) SLE patients had at least one cardiac abnormality ( $p<0.0001-\mathrm{RR} 19 ;$ OR 41.8; 95\% CI 5.1-342). The predominant valve dysfunctions observed were mitral (21\%) and tricuspid (18\%) regurgitation, aortic regurgitation (12\%), pericardial effusion (15\%), and left atrial enlargement (12\%). Echocardiographic abnormalities were more common in aPLs positive than in aPLs negative patients $(69.6 \%$ vs $27.3 \%)(p=0.02$; RR 2.5 ; OR 6.1 ; 95\% CI 1.2-30.1). LA was detected in 14 patients $(44.1 \%)$ and negative in all controls $(p<0.0001)$. IgM and IgG aCL were present in eight (23.5\%) and in 17 (50\%) SLE patients, respectively, and were absent in the control group $(p<0.01)$. LA and/or aCL (low and medium-high titer) positivity was not significantly associated with the presence of TEE abnormalities. Anti-phosphatidic acid (aPA)-IgG antibodies were found positive in 12 cases $(35.3 \%)$ and were associated with left atrial enlargement $(p=0.01)$ and increased interventricular septum thickness $(p<0.05)$. The presence of anti-phosphatidyl serine (aPS)-IgG antibodies was detected in 14 cases $(41.2 \%)$ and was associated with left atrial enlargement $(p<0.05)$. Anti-phosphatidyl inositol (aPI)-IgG antibodies, positive in 13 cases (38.2\%), were statistically associated with left atrial enlargement $(p=0.01)$ and increased interventricular septum thickness $(p<0.05)$. IgM and IgG anti-b2GPI were present in six (17.6\%) and in 12 SLE patients (35.3\%), respectively, with no statistical association with echocardiographic findings.

Continuing addressing the topic about the accuracy of tools to measure the valvulopathy as an outcome, a randomized controlled study conducted by Roldan et al. [49] analyzed the accuracy of TEE compared to the accuracy of transthoracic echocardiography (TTE) for detection of valvular heart disease and masses in SLE patients. They performed transesophageal echocardiograms in: (1) 54 patients with SLE, 22 of them with aPL (Group I) and 32 without aPL (Group II); (2) ten patients with APS (Group III); and (3) in 35 normal individuals (Group IV). The patients in groups I and III had similar isotypes and concentrations of antibodies. LS vegetations were observed in $41 \%$ of the patients in Group I, $25 \%$ of II, $10 \%$ of III, and in no patient in IV (I or II vs. IV, $p<0.002$ ). Valvular regurgitation was found in $64 \%$ of the patients in Group I, $59 \%$ of II, $10 \%$ of III, and in $20 \%$ of IV (I or II vs. III or IV, $p<0.006$ ). This study demonstrated that in SLE, in the presence or absence of aPL, had similar prevalence and severity of valvular disease. 
Despite these results, it is suggested that the presence of aPL plays a role in the pathogenesis of the cardiac lesions in patients with SLE, as explored in two systematic reviews [50] and [51]. Mattos et al. carried out the first one in order to better understand this point in 2011. Twenty articles were found, which evaluated the association between the presence of aPL and valvulopathy. Thirteen of these studies evaluated the association of aPL with the LS lesion. Of the 20 articles, 15 demonstrated a positive association between aPL and valvular lesions, whereas five articles demonstrated that there was no association. Evidence of an association between the presence of aPL and valvular lesion was demonstrated in most of the studies. Nevertheless, the possible role of these antibodies in the etiopathogenesis of the heart valve lesion has not yet been proved. Zuily et al. conducted a systematic review and meta-analysis to estimate the risk for HVD and/or Libman-Sacks' endocarditis associated with aPL in SLE patients. They included 23 studies (15 cross-sectional, 7 cohort and one case-control) with 1656 SLE patients (668 with aPL, and 988 without aPL). HVD was present in 43\% of aPL positive patients and in $22 \%$ of aPL negative patients. The OR for valvulopathy in aPL positive patients was 3.13 (95\% CI 2.3-4.24) and for Libman-Sacks' endocarditis 3.51 (95\% CI 1.93-6.38). The risk of valvulopathy depending on aPL subtypes was the highest for LA at 5.88 (95\% CI 2.92-11.84) and for aCL IgG at 5.63 (95\% CI 3.53-8.97). Although they were SRs, for the purpose of this analysis, they were indirectness, as they analyzed patients with SLE, which down the GRADE. Despite this, they corroborate the assumption that aPL plays a role in the pathogenesis of the cardiac lesions in patients with APS.

\subsubsection{Overall quality of evidence and recommendations}

We found studies with robust design to address the issues included in this topic, including cohort studies, RCT and two systematic reviews that allowed us to start the analysis for the body of evidence as GRADE high. The frequency of the manifestation, clinical features in long term and consequence for clinical decision-making was explored in the studies analyzed. Some studies included SLE patients that aimed to discriminate its peculiarities in the context of APS, as it is categorized as critical for decision-making and will require different decisions for the practitioner. There is evidence that the prevalence of heart valve disease is very high in APS, the lesion according to definition is characteristic and even though the pathogenic mechanisms are not known, there is evidence that aPL have a role in these lesions.

\subsection{Livedo reticularis}

Specific question: "In patients with APS $(P)$, is livedo reticularis, specifically livedo racemosa combined by positive aPL $(I / C)$, associated with APS classification, leading to earlier and most adequate treatment intervention to prevent thrombotic events $(O)$ '"?

PICO: P — APS; I/C — livedo reticularis, specifically livedo racemosa combined by positive aPL; $\mathrm{O}$ the occurrence of recurrent thrombotic events, including serious life threatening event.

\subsubsection{Patient-important outcome}

Despite the fact that the dermatologic literature contains only reports of small series or isolated cases of cutaneous manifestations in APS, livedo reticularis has been considered as the most frequently observed 
dermatologic manifestation (25.5\%), with a similar prevalence in PAPS (31\%) and SLE-related APS (20\%). It was a presenting manifestation in $17.5 \%$ of cases. Regardless of the type of skin involvement, it is important to remove or reduce other risk factors for thrombosis or arterial wall lesions. For example, patients are advised to stop smoking, and women are counseled against the use of estrogen-containing pills. The potential benefit of treatment with statins or angiotensin-converting enzyme inhibitors needs to be determined, especially in patients with livedo. With the assumption that livedo reticularis can be associated with thrombotic events, the patient-important outcome was defined as the occurrence of recurrent thrombotic events, including serious life threatening event. It was categorized as important but not critical for decision-making.

\subsubsection{Findings}

There were five studies and three were cohort studies. The outcome's classification of each study had directness in all of them. The patient-important outcome was the occurrence of recurrent thrombotic events, including serious life threatening events which were categorized as important but not critical for decision-making.

Two studies evaluated the frequency of cutaneous lesions in patients with APS[52] and [53]. Diógenes included 21 patients with positive aPL profile without APS and found that $40 \%$ presented cutaneous features, of which $7 \%$ had livedo reticularis. Francès et al. showed a $49 \%$ frequency of dermatologic manifestation (livedo reticularis in 25\%). Also, a decreased frequency of venous thrombosis was observed in patients with livedo reticularis (OR $0.2,95 \%$ CI $0.1-0.5$ ). Additionally of this research question, Francès et al. compared cutaneous manifestations with pregnancy morbidity, CNS and cardiac manifestation in order to investigate the risk to develop these manifestations. Significant livedo reticularis associations were verified in: (1) cerebral or ocular ischemic arterial events (OR 10.8, 95\% CI 5.2-22.5); (2) seizures (OR 6.5, 95\% CI 2.6-16); (3) all arterial events (OR 6.0, 95\% CI 2.9-12.6); (4) heart valve abnormalities by TEE (OR 7.3, 95\% CI 3.6-14.7); and (5) arterial systemic hypertension $(>160 \times 90 \mathrm{~mm} \mathrm{Hg})($ OR 2.9, 95\% CI 1.5-5.7). Toubi et al. described livedo reticularis in 50 of 308 patients with APS (16\%) and also found significant associations between livedo reticularis and CVA $(p=0.01)$; migraines $(p=0.002)$; and epilepsy $(p=0.02)$ [54].

\subsubsection{Overall quality of evidence and recommendations}

The overall quality of evidence to support the assumption that livedo reticularis - specifically livedo racemosa combined with positive aPL — is associated with APS classification in five cohorts started as GRADE high. However, in those that assessed frequencies, CI 95\% and $p$-value were not shown, representing inconsistencies of results. These aspects decrease the quality of the body of evidence to GRADE moderate. Based on this, our recommendation reflects a belief that livedo reticularis should be evaluated in further studies to better support this assumption. 


\subsection{Migraine}

Specific question: "In patients with APS $(P)$, is migraine $(I)$ associated with APS classification, leading to earlier and most adequate treatment intervention to prevent thrombotic events $(O)$ "'?

PICO: P — APS; I/C - migraine; O - thrombotic events.

Patient-important outcome: thrombotic events.

Preliminary relative importance: important but not critical for decision-making.

\subsubsection{Patient-important outcomes}

Migraine has been described as a common in patients with APS, and although it sometimes is responsive only to anticoagulants, any causal relation has not been proven.

\subsubsection{Findings}

Migraine has been described as a common symptom in patients with APS, and although it sometimes is responsive only to anticoagulants, the causal relation is an issue about which different groups of researches do not have a common opinion. With this conflict in mind a systematic review was performed.

There were 10 studies included in this analysis. Among clinical features of 1.000 APS patients from 13 European countries, 53.1\% with PAPS, 36.2\% with APS associated to SLE and 10.9\% with APS associated with other diseases, migraine was mainly described on females $-23 \%$ vs. $12 \%$ [8]. These patients were followed for five years. Migraine was the most frequent neurologic manifestation, found in $20.2 \%$ [20]. Krause et al. evaluated 246 APS patients, age ranging from 19 to 75 years old (61.8\% PAPS). Migraine occurred in $19.5 \%$ of these patients [55]. In a retrospective analysis of 14 years, from 128 patients with PAPS at first, Gómez-Puerta et al. observed who developed SLE or other autoimmune disease. The average age was $42 \pm 12$, with a follow-up of $9 \pm 3$ years. In the end, $86 \%$ of the patients remained with the PAPS diagnosis. Migraine was one of the main manifestations reported, encountered in $40 \%$ of patients [56]. As presented in the cardiac section, Krause et al. (2005) also evaluated the connection between cardiac valvar lesion and CNS involvement in 284 APS patients (55.9\% PAPS). Significant clusters were found between valvar vegetations and epilepsy $(p<0.002)$ and between valvar thickening/dysfunction - as well as overall valvar abnormalities (vegetations and/or valvar dysfunction) - and migraine ( $p=0.002$ for both). A borderline association was found between valve vegetation and migraine $(p=0.09$ ). The migraine prevalence on the group as a whole was $18 \%$, and this symptom was mostly found in the PAPS group (20.8\% vs. $14.4 \%$, respectively).

Garcia-Carrasco et al. evaluated Latin American APS patients (57\% PAPS, 35\% APS associated with lupus, $8 \%$ APS associated with other diseases). Migraine was present in $25 \%$ of patients at the time of diagnosis [57], being the most frequent neurologic complaint. In comparison with the European Group, migraine was more frequent in the Latin Americans (OR 2.16, IC 95\% 1.33-3.49, $p=0.001$ ). A study including 100 Latin American Mestizos (from Colombia, Ecuador and Mexico), all fulfilling PAPS criteria, compared their clinical and immunological manifestations with 538 Caucasians with PAPS 
(from the Euro-phospholipid cohort). Migraine prevalence in both groups was 18\%, and it was the most frequent neurologic complaint of the former [58].

As genetic factors may influence migraine development, Williams et al. evaluated the prevalence of aPL in migraine-discordant monozygotic twins. There was no statistically significant difference between the groups with or without migraine, or between patients of the migraine group as for the type of aPL present [59].

Cavestro et al. evaluated the prevalence of aPL in a random cohort of 284 migraine patients with or without aura, and compared them with 225 sex and age-matched controls. Positivity for at least one aPL test (LA, aCL IgG or anti-b2GPI IgG) was detected and confirmed in $33(12 \%)$ patients and in 7 (3\%) controls (OR 4.08; CI 1.77-9.39; $p=0.0004$ ). The most prevalent antibody was anti-b2GPI IgG, present in $80.8 \%$ of patients and in $2.5 \%$ of controls (OR 3.51, CI 1.41-8.71, $p=0.004$ ) [60].

The Serbian cohort previously cited (Stojanovich et al.) [19] analyzed 374 Caucasian patients, 69.5\% PAPS, 30.5\% APS associated with SLE. They investigated the association between non-criteria APS manifestations and aPL type and titers. They found migraine to be the most frequent complaint (70 patients, 26.9\%) in the PAPS group, occurring mainly in patients with IgM aCL ( $p=0.017)$. The statistical analysis of the antibodies showed a connection between migraine occurrence and LA absence $(p=0.014)$. In APS associated with SLE, migraine was found in $32.5 \%$ of patients.

\subsubsection{Overall quality of evidence and recommendations}

Migraine is the most frequent CNS complaint in PAPS patients, and there is no association with a specific aPL. This feature is more prevalent among females, and it can be associated with cardiac vegetation. The literature does not show that this comorbidity is more prevalent in PAPS then in the general population. This limitation is due to the design of these studies (most of them are observational studies), and due to some (4/10) not using International Headache Society Criteria. In addition, the studies have not included brain imaging (if stroke were shown, for example, the stroke would explain the association with aPL). We do not recommend treatment with anticoagulants if migraine is the unique symptom. Well-designed randomized controlled trials are urgently needed to verify the possible association between refractory migraine or migraine like and PAPS. The final evaluation of these efforts was GRADE very low for the body of evidence.

The outcome of this analysis does not support the assumption that migraine and APS are causally associated. We strongly recommend that further studies applying clinical and laboratory diagnostic criteria both for headache, brain imaging and for APS are needed in order to study a possible association. It is important to emphasize that additional work is also required to assess whether antithrombotic or other drugs targeting aPL may be advantageous in patient without a history of thrombosis.

\subsection{Chorea, seizures and longitudinal myelitis}

Specific question (chorea, longitudinal myelitis and seizures): "In patients with APS $(P)$, is chorea $(I)$ associated with APS diagnose, leading to earlier and most adequate treatment intervention to prevent thrombotic events or other neurologic complications $(O)$ "'? 


\subsubsection{Patient-important outcomes}

A spectrum of neuropsychiatric manifestations, including stroke and TIA are accepted as "thrombotic", but other inflammatory manifestations may occur in the neurologic system. Many other neuropsychiatric manifestations, including chronic headache, cognitive dysfunction, psychosis, depression and multiple sclerosis-like-illness, have also been reported with aPL positivity.

\subsubsection{Findings}

For the purpose of this paper, the experts considered chorea, longitudinal myelitis and seizures separately. The late Ronald Asherson et al. first considered the association between chorea and SLE in 1987, when they described a case series. They reported twelve patients with chorea from a population of 500 patients with SLE and "lupus-like" disease, including a clinical review, time relationship of the chorea to the systemic illness and other neurologic manifestations. Chorea appeared early in the course in most patients, but the development of cerebral infarctions or TIAs occurred subsequently in seven out of nine patients with aPL [61]. Some questions derived from these observations and guided subsequent studies. Chorea is a rare manifestation of APS. The Euro-Phospholipid Project has estimated that the prevalence of chorea in APS is of $1.3 \%$. Chorea has been described in association with primary APS in a number of patients, published as case reports and some case series [62]. Orzechowski et al. [63] and Reiner et al. [64] also observed association between APS and chorea.

The association of seizures with aPL antibodies in SLE or non-SLE has been widely studied, with conflicting results. Verrot et al. [65]; Cimaz et al. [66]; Peltola et al. [67]; and the case-control series of Liimatainen et al. [68] described seizures in non-SLE patients with increased aPL. On the other hand, seizures in SLE patients were described with positive results by Herranz et al. [69]; Mackworth-Young and Hughes [70], Toubi et al. [71]; Liou et al. [72]; Kavassa et al. [73]; Mok et al. [74]; Sanna et al. [75]; Appenzeller et al. [76] and Mikdashi and Handwerger [77]. Sachse et al. [78] and Formiga et al. [79] described negative associations. Additionally, if SLE patients with strokes are excluded, there is no association of seizure with lupus anticoagulant (Table 1).

Longitudinal myelitis is an acute inflammatory process that affects a longitudinal segment of the spinal cord. D'Cruz et al. reported 15 patients in whom longitudinal myelitis was the initial manifestation of SLE or of lupus-like disease [80]. Of those patients, $73 \%$ tested positive for aPL, which provides further evidence of a strong correlation between longitudinal myelitis and aPL positivity. The pathogenic mechanism seems to involve a direct interaction between aPL and spinal cord phospholipids rather than a thrombotic process leading to ischaemia, although no strong correlation has been made between these manifestations and APS. Birnbaum et al. [81] described distinct subtypes of myelitis in systemic lupus erythematosus. Eleven patients presented with signs of gray matter dysfunction (i.e., flaccidity and hyporeflexia), whereas 11 patients presented with signs of white matter dysfunction (i.e., spasticity and hyperreflexia). Patients with gray matter dysfunction were more likely to have irreversible paraplegia ( $p<0.01)$, despite presenting with a monophasic versus polyphasic course $(p=0.01)$, higher levels of SLE activity (mean SLE Disease Activity Index 9.8 versus $2.0 ; p=0.01$ ), and a cerebrospinal fluid profile indistinguishable from bacterial meningitis. Prior to irreversible paraplegia, these patients 
presented with prodromes of fever and urinary retention, but were misdiagnosed by physicians of different specialties as having urinary tract infections. Patients with white matter dysfunction were more likely to meet criteria for neuromyelitis optica $(p=0.04)$ and were also more likely to have aPL (lupus anticoagulant) $(p=0.01)$. These findings indicate that SLE myelitis encompasses two distinct and previously unrecognized syndromes that can be distinguished clinically by gray matter versus white matter findings. Recognition of fever and urinary retention as prodromes of irreversible paraplegia may allow earlier diagnosis and treatment in SLE patients presenting with gray matter findings.

\subsubsection{Overall quality of evidence and recommendations}

There are neurologic, cardiac and hematologic manifestations of aPL. The mechanism is inflammation, as they respond to corticosteroids. We should stop calling them "non-criteria" and re-think what APS really means. All future studies of seizure with aPL must include brain imaging and adjust for the occurrence of stroke as the cause of seizure.

\subsection{The so-called seronegative APS (SN-APS)}

Specific question 1: In patients with clinical features suggestive of APS which are persistently negative for the routinely aPL (aCL, anti-b2GPI, and LA) what is the advantage to classify them as seronegative APS (SN-APS)? Does this lead to earlier and most adequate treatment intervention to prevent further thrombotic events?

$P O-P$-APS; $O$ - thrombotic events.

Specific question 2: In real clinical practice $(P)$, how often are the discrepancies between aPL detection and clinical APS expression $(O)$ ?

Patient-important outcome: peculiar thrombotic events.

Surrogate: so-called seronegative APS (SN-APS).

Preliminary relative importance: critical for decision-making.

\subsubsection{Patient-important outcomes}

The notion of a clinical syndrome, where classical clinical manifestations (Sapporo/Sydney criteria) allied to some of the most commonly associated features (non-criteria manifestations) could suggest a hypercoagulability state including APS, even though none of the aPL antibodies of the classification criteria is found, seems plausible[82] and [83]. This notion was raised based on clinical observations, and first published in 2003 by Hughes and Khamashta. Therefore, the term 'seronegative APS' (SN-APS) has been coined to include these patients with clinical features suggestive of APS who are persistently negative for aPL and other thrombophilias [84]. The importance of this observation is due to the fact that these patients will require proper counseling (to reduce the thrombotic risk) and sometimes also treatment, because they are prone to recurrent thromboses and even catastrophic events. The other relevant dimension of this debate is related to the factors that can suggest the presence of SN-APS to the 
characteristics of endothelial molecules specifically recognized by serum autoantibodies to define SNAPS[85].

There was a controversy among the task force members, which discussed that despite the robust association between autoantibodies and APS, some patients remain persistently negative for disease specific autoantibodies. On the other hand, though the prevalence of seronegative autoimmune diseases is low, they may represent a practical problem because they are often difficult cases and should not remain untreated. When there is a clinical picture suggestive of APS, and transient negativity due to recent thrombosis and antibody consumption, as well as other causes of thrombosis are excluded, it is important to consider a clinical diagnostic of APS. Therefore, it was proposed for these cases the so-called term "seronegative APS (SN-APS)" when the classical tests are not detected, but there may be isolated IgA reactivity or to other test (anti-PS/PT, anti-D1, anti-annexin $\mathrm{V}, \mathrm{aCL} /$ vimentin or others that were discussed by the laboratory Task Force) that is not included in the criteria, and not yet standardized and widely available [86], [87] and [88].

The dimensions on the existence of SN-APS concepts may be summarized on clinical observations; challenges on prevalence studies and challenges on laboratory studies. The importance of this debate is directly linked to the consequences for patients, as the decisions required will — or not — be influenced by it. Taking these points into account, this outcome is considered as important and very critical for decision making, as the consequences of a misdiagnosis can be bound to serious and life threatening conditions.

\subsubsection{Findings}

There were seven studies included on this analysis: two case reports, one case-control, three cohort studies, and one non-systematic literature review. The two case-reports are presented here in order to provoke the discussion about the consequence of this diagnosis for the clinical practice, but were not included in the analyses. As patient important outcomes are categorized by how critical is the consequence of the misdiagnosis - and consequently, the mistreatment for the patient, these cases can contribute for the constructs of the questions. Also, we included a literature review published by Cervera et al. that present the arguments for and against the existence of SN-APS [89].

In this cited literature review, the authors discussed that in favor of this are the fact that new emerging aPL assays could improve our ability in diagnosing APS. Transient or false negative aPL assay and other genetic or acquired pro-thrombotic conditions have to be considered and can further complicate this issue. On the other hand, one of the most critical issues is that most laboratories test only IgG and IgM aCL (few may be able to test for IgA) and LA, and only a few of them test anti-b2GPI (primarily IgG isotype). Furthermore, the currently available assays cannot detect all potential aPL, and the new specificities, recently identified, are tested in only a few research laboratories. Another important issue is that transient aPL seronegativity which may be due to different causes, such as nephrotic syndrome, treatment with corticosteroids, immunosuppressants, or antibody consumption during thrombotic event may take a longer follow-up and persistent re-testing for identification. 
There is only one paper comparing SNAPS and seropositive APS [90]. The authors retrospectively assessed clinical manifestations of APS in 154 patients: 87 patients with seropositive APS and 67 patients with thrombosis and/or pregnancy morbidity persistently negative for aPL and presenting with at least two additional non-criteria manifestations of APS (the so-called 'seronegative APS', SN-APS). The results showed no significant differences in the frequency of thrombotic events or obstetric morbidity in patients with SN-APS versus patients with seropositive APS: deep vein thrombosis $(31.4 \%$ vs. 31.0\%), pulmonary embolism $(23.8 \%$ vs. $28.7 \%)$, stroke (14.9\% vs. $17.2 \%)$, transient ischaemic attack (11.9\% vs. $10.3 \%)$, early spontaneous abortions (67.1\% vs. $52.1 \%)$, stillbirths (62.5\% vs. $59.4 \%)$, prematurity ( $28.1 \%$ vs. $21.7 \%$ ) or pre-eclampsia (28.1\% vs. $23.1 \%)$. Based on this, the authors suggest that clinical management in patients with APS should not be based only on the presence of conventional aPL. The understanding of the prevalence of aPL in patients with SLE in addition to the knowledge of the association with thrombosis, association with non-thrombotic disease manifestations, and lack of association with atherosclerosis were the issues explored by the review of Hopkins' Lupus Cohort [91]. In spite of its indirectness for this discussion here (it analyzed SLE patients), these findings can contribute to go beyond this debate. The aPL detection as common in patients in the Hopkins' Lupus Cohort: 47\% have aCL, 32.5\% anti-b2GPI and 26\% LA (by dRVVT confirmatory testing) is an important finding to this specific debate; and the fact that aPL were not associated with atherosclerosis.

In addition of this study, anti-prothrombin antibodies (aPT) have been reported as the sole antibodies detected in a few patients with SLE and a history of thrombosis but persistently negative for aCL or LA [92]. Also, it has been shown that antibodies directed to the lyso (bis) phosphatidic acid (aLBPA) may represent a marker of APS showing similar sensitivity and specificity compared to anti-b2GPI. In addition, aLBPA are associated strongly with the presence of LA. Conti investigated the clinical usefulness of thin-layer chromatography (TLC) immunostaining in detecting serum aPL in patients presenting clinical features of SN-APS. Sera from 36 patients with SN-APS, 19 patients with APS, 18 with SLE, 20 anti-hepatitis C virus-positive subjects and 32 healthy controls were examined for aPL using TLC immunostaining [93].

The outcomes assessed on this cohort were venous and/or arterial thrombosis and pregnancy morbidity, as stated in the classification criteria for definite APS. TLC immunostaining showed aPL in 58.3\% of SNAPS patients: aCL in 47.2\%, anti-lyso (bis) phosphatidic acid in $41.7 \%$ and anti-

phosphatidylethanolamine in $30.5 \%$. Six of 36 patients showed anti-annexin II. Also, they found a statistically significant correlation was found between vascular thrombosis (arterial and/or venous) and pregnancy morbidity in SN-APS ( $p<0.0001$ ); and a statistically significant correlation was found among aCL, aLBPA and aPE positivity $(p<0.02)$. The most relevant finding of this study is that TLC immunostaining could potentially identify the presence of aPL in patients with clinical features suggestive of APS not ascertained by traditional tests for aPL, and such identification could have a major impact on the prognosis and therapeutic approach.

\subsubsection{Overall quality of evidence and recommendations}

The existence of SN-APS is debated among the task force members, as the concept of the "so-called" Seronegative APS is still hotly debated. Even though, some considered that it is important to be evaluated 
and that it's prognostic significance must be elucidated. This scenario could represent a trade-off between the low quality of evidence (GRADE low) and a critical for decision-making outcome.

\section{Discussion}

A series of clinical features, that are not included in the classification criteria, but are recognized to be related to APS have been named non-classical or non-criteria clinical manifestations. An International Task Force reviewed those that were considered more clinically relevant, in addition to SN-APS, that although it is not consensus among the task force members, some considered it important to be investigated. We used the GRADE system to evaluate the medical literature on the relevance of considering the inclusion of each feature as part of a revised classification criterion, which will aid to recognize the broader spectrum of the clinical picture of APS. These clinical characteristics are not exclusively related to pathogenic events clearly involving thromboses, but occur also when thrombosis is not evident. Their relationship with aPL and the classical features of APS urges us to give these features their proper value in diagnostic work-up, in terms of defining prognostic and morbidity correlations and optimally provide diagnostic investigation, as well as, treatment recommendation when appropriate in order to improve patient outcome.

To start making recommendations regarding APS criteria, the GRADE system considers the balance of patient-important outcomes, the overall quality of the evidence for each outcome, the uncertainty about values and preferences associated with the diagnostic strategies and the presumed patient-important outcomes, and cost. We analyzed the outcomes presented by the APS experts that were discussed during the task force. Then, we categorized their importance to patient care, and analyzed the overall quality of evidence. Finally, we presented the strength to consider each one as part of APS diagnostic criteria (Table 2).

Analyzing SVT, there is a low overall quality of evidence to support the suggestion that SVT is due to aPL/APS, unless there are other features of APS.

Thrombocytopenia is critical for decision-making. Once the most common causes are ruled out, aPL should be checked even in the absence of other features that are characteristic of APS. The overall quality of evidence for this finding was low. In general, aPL related thrombocytopenia is mild or moderate and the risk of bleeding is minimal even with very low counts. Data in the literature indicate that thrombosis risk is increased in these patients.

Kidney failure or deaths due to kidney failure are the worst consequences of APS nephropathy. These are the patient-important outcome here and they are critical for decision-making. Based on them, and the moderate quality of the overall evidence, there is strong support to have the diagnosis confirmed by histopathology, taking into consideration that TMA is a surrogate for the patient-important outcome. Patient-important outcomes linked to heart valve disease related to aPL were categorized as very critical for decision making. Taking this, together with the moderate quality of the body of the evidence, there is moderate support to recommend its inclusion as part of APS classification criteria. 
Livedo reticularis can be associated with the occurrence of thrombosis and this outcome was defined as important but not critical for decision-making. There is a suggestion, with moderate quality of evidence that it can be included as part of diagnostic criteria. In the same way, migraine can be associated with thrombotic events, but this outcome was categorized as low importance for decision-making. Chorea, myelitis and seizures were analyzed separately in this task force. Their consequences for decision-making were categorized as very critical for decision making, which support, based on the overall quality of evidence, that chorea and longitudinal myelitis should be included as part of APS criteria but not seizures. Regarding SN-APS, the consequences for decision-making, are categorized as very critical for decisionmaking. Despite this, the overall quality of evidence was low, as they included editorials, non-systematic review, and case reports, which downgrade the body of evidence. Therefore, considering the trade-off between misdiagnosis and the risk of future events, it is strongly recommended to conduct further studies to determine its real significance.

\section{Conclusion}

The purpose of this task force was to critically analyze the so-called non-criteria manifestations of APS to support their inclusion as APS classification criteria. Classification criteria are important for including homogeneous groups of patients in observational as well as in therapeutic studies. The case has been made for including thrombocytopenia, renal microangiopathy, heart valve disease and two neurological manifestations (chorea and longitudinal myelitis) as classification criteria. However such a decision should be carefully balanced because a change of classification criteria will lead to consider different subsets of patients as APS patients. Thus a consensus not only of the authors of the present paper but of all experts in the field is needed. Such an effort is currently underway for the next antiphospholipid meeting in Istanbul and the present review will be a basis for classification revision.

\section{Take-home messages}

- The purpose of this task force was to critically analyze the so-called non-criteria manifestations of APS to support their inclusion as APS classification criteria.

- Using GRADE system, we found that overall quality of evidence was very low for migraine and seizures,; low for superficial venous thrombosis, thrombocytopenia, chorea, longitudinal myelitis and the so-called seronegative APS, and moderate for APS nephropathy, heart valve lesions and livedo reticularis.

- A critical redefinition of an APS gold standard will be the next steps and it can include data from the APS ACTION registry that will include not only current APS patients but also those with antiphospholipid antibodies not meeting current classification criteria. 


\section{References}

[1] Wilson WA, Gharavi AE, Koike T, et al. International consensus statement on preliminary classification criteria for definite antiphospholipid syndrome: report of an international workshop. Arthritis Rheum 1999;42:1309-11.

[2] Guyatt GH, Oxman AD, Vist GE, et al. GRADE: an emerging consensus on rating quality of evidence and strength of recommendations. BMJ 2008;336(7650):924-6.

[3] Mendes de Abreu M, Wahl D, Levy RA. The use of GRADE system on autoimmune diseases: challenges for shared decision-making. Lupus 2014;23(12):1292-4.

[4] Guyatt GH, Oxman AD, Schünemann HJ, et al. GRADE guidelines: a new series of articles in the Journal of Clinical Epidemiology. J Clin Epidemiol 2011;64:380-2.

[5] de Jesus GR, Agmon-Levin N, Andrade CA, et al. 14th International Congress on Antiphospholipid Antibodies Task Force Report on Obstetric Antiphospholipid Syndrome. Autoimmun Rev 2014;13(8):795-813.

[6] Erkan D, Aguiar CL, Andrade D, et al. 14th International Congress on Antiphospholipid Antibodies: task force report on antiphospholipid syndrome treatment trends. Autoimmun Rev 2014;13(6):685-96.

[7] Cervera R, Rodríguez-Pintó I, Colafrancesco S, et al. 14th International Congress on Antiphospholipid Antibodies Task Force Report on Catastrophic Antiphospholipid Syndrome. Autoimmun Rev 2014;13(7):699-707.

[8] Arnaud L, Mathian A, Devilliers H, Ruffatti A, Tektonidou M, Forastiero R, et al. Patient-level analysis of five international cohorts further confirms the efficacy of aspirin for the primary prevention of thrombosis in patients with antiphospholipid antibodies. Autoimmun Rev 2014 Oct;22(14):00240-7 (pii: S1568-9972).

[9] Cervera R, Piette JC, Font J, et al. Antiphospholipid syndrome: clinical and immunologic manifestations and patterns of disease expression in a cohort of 1,000 patients. Arthritis Rheum 2002;46:1019-27.

[10] 0 Zuily S, Regnault V, Guillemin F, Kaminsky P, Rat AC, Lecompte T, et al. Superficial vein thrombosis, thrombin generation and activated protein $\mathrm{C}$ resistance as predictors of thromboembolic events in lupus and antiphospholipid patients. A prospective cohort study. Thromb Res 2013;132:e1-7. [11] Leizorovicz A, Becker F, Buchmüller A, et al. Clinical relevance of symptomatic superficial-vein thrombosis extension: lessons from the CALISTO study. Blood 2013;122(10):1724-9.

[12] Reynaud Q, Lega JC, Mismetti P, Chapelle C, Wahl D, Cathébras P, et al. Risk of venous and arterial thrombosis according to type of antiphospholipid antibodies in adults without systemic lupus erythematosus: a systematic review and meta-analysis. Autoimmun Rev 2014;13(6):595-608.

[13] Yang YJ, Yun GW, Song IC, et al. Clinical implications of elevated antiphospholipid antibodies in adult patients with primary immune thrombocytopenia. Korean J Intern Med 2011;26(4):449-54.

[14] Kim KJ, Baek IW, Yoon CH, et al. Thrombotic risk in patients with immune thrombocytopenia and its association with antiphospholipid antibodies. Br J Haematol 2013; 161(5):706-14.

[15] Dasanu CA, Codreanu I. Isolated thrombocytopenia: should we routinely screen for antiphospholipid antibodies? Conn Med 2011;75(5):281-4. 
[16] Diz-Kucukkaya R, Hacihanefioglu A, Yenerel M, et al. Antiphospholipid antibodies and antiphospholipid syndrome in patients presenting with immune thrombocytopenic purpura: a prospective cohort study. Blood 2001;98:1760-4.

[17] Stasi R, Stipa E, Masi M, et al. Prevalence and clinical significance of elevated antiphospholipid antibodies in patients with idiopathic thrombocytopenic purpura. Blood 1994;84:4203-8.

[18] Cervera R, Boffa MC, Khamashta MA, Hughes GR. The Euro-Phospholipid project: epidemiology of the antiphospholipid syndrome in Europe. Lupus 2009;18(10): 889-93.

[19] Italian Registry of Antiphospholipid Antibodies (IR-APA). Thrombosis and thrombocytopenia in antiphospholipid syndrome (idiopathic and secondary to SLE): first report from the Italian Registry. Haematologica 1993;78(5):313-8.

[20] Cuadrado MJ, Mujic F, Muñoz E, et al. Thrombocytopenia in the antiphospholipid syndrome. Ann Rheum Dis 1997;56(3):194-6. [21] 1 Stojanovich L, Kontic M, Djokovic A, et al. Association between systemic noncriteria APS manifestations and antibody type and level: results from the Serbian national cohort study. Clin Exp Rheumatol 2013;31(2) (234-4).

[22] Comellas-Kirkerup L, Comellas-Kirkerup L, Hernández-Molina G, Cabral AR. Antiphospholipidassociated thrombocytopenia or autoimmune hemolytic anemia in patients with or without definite primary antiphospholipid syndrome according to the Sapporo revised classification criteria: a 6-year follow-up study. Blood 2010;116(16) (3058-6).

[23] Krause I, Blank M, Fraser A, et al. The association of thrombocytopenia with systemic manifestations in the antiphospholipid syndrome. Immunobiology 2005;210: 749-54.

[24] Erkan D, Lockshin MD, APS ACTION members. APS ACTION - AntiPhospholipid Syndrome Alliance For Clinical Trials and International Networking. Lupus 2012; 21(7):695-8.

[25] Levy RA, Jesús GRR. APS ACTION in Brazil. Braz J Rheumatol 2012;52(5):813-4.

[26] This point was discussed by email with Professor A Holger J Schünemann, who provided elements for a more assertive judgment about the quality of evidence in the case of APS disease.

[27] Cervera R, Tektonidou MG, Espinosa G, et al. Task Force on Catastrophic Antiphospholipid Syndrome (APS) and Non-criteria APS Manifestations (I): catastrophic APS, APS nephropathy and heart valve lesions. Lupus 2011;20(2):165-7.

[28] Nangaku M, Alpers CE, Pippin J, et al. CD59 protects glomerular endothelial cells from immunemediated thrombotic microangiopathy in rats. J Am Soc Nephrol 1998;9(4):590-7.

[29] Seshan SV, Franzke CW, Redecha P, et al. Role of tissue factor in a mouse model of thrombotic microangiopathy induced by antiphospholipid antibodies. Blood 2009; 114(8):1675-83.

[30] Wu LH, Yu F, Tan Y, et al. Inclusion of renal vascular lesions in the 2003 ISN/RPS system for classifying lupus nephritis improves renal outcome predictions. Kidney Int 2013;83(4):715-23.

[31] Amigo MC, Garcia-Torres R, Robles M, et al. Renal involvement in primary antiphospholipid syndrome. J Rheumatol 1992;19:1181-5.

[32] Nochy D, Daugas E, Droz D, et al. The intrarenal vascular lesions associated with primary antiphospholipid syndrome. J Am Soc Nephrol 1999;10:507-18.

[33] Daugas E, Nochy D, du LT Huong, et al. Antiphospholipid syndrome nephropathy in systemic lupus erythematosus. J Am Soc Nephrol 2002;13:42-52. 
[34] Tektonidou MG, Sotsiou F, Nakopoulou L, et al. Antiphospholipid syndrome nephropathy in patients with systemic lupus erythematosus and antiphospholipid antibodies: prevalence, clinical associations, and long-term outcome. Arthritis Rheum 2004;50(8):7-2569.

[35] Glueck HI, Kant KS, Weiss MA, et al. Thrombosis in systemic lupus erythematosus. Relation to the presence of circulating anticoagulants. Arch Intern Med 1985;145:1389-95.

[36] Kant KS, Pollak VE, Weiss MA, et al. Glomerular thrombosis in systemic lupus erythematosus. Prevalence and significance. Medicine 1981;60:71-86.

[37] Tektonidou MG, Sotsiou F, Moutsopoulos HM. Antiphospholipid syndrome (APS) nephropathy in catastrophic, primary and systemic lupus erythematosus-related APS. J Rheumatol 2008;35:1983-8. [38] Song D, Wu LH, Wang FM, et al. The spectrum of thrombotic microangiopathy in lupus nephritis. Arthritis Res Ther 2013;15:R1-12.

[39] Denas G, Jose SP, Bracco A, Zoppellaro G, Pengo V. Antiphospholipid syndrome and the heart: a case series and literature review. Autoimmun Rev 2014;14:4-00275 (pii: S1568-9972).

[40] Petri M. Classification criteria for antiphospholipid syndrome: the case for cardiac valvular. J Rheumatol 2004;31:2329-30.

[41] Hollan I, Meroni PL, Ahearn JM, Cohen Tervaert JW, Curran S, Goodyear CS, et al. Cardiovascular disease in autoimmune rheumatic diseases. Autoimmun Rev 2013; 12(10):1004-15.

[42] Espinola-Zavaleta N, Amigo MC, Vargas-Barròn J, et al. Two and three-dimensional echocardiography in primary antiphospholipid syndrome: misdiagnosis as rheumatic valve disease. Lupus 2001;10:511-3.

[43] Bouillanne O, Millaire A, de Groote P, et al. Prevalence and clinical significance of antiphospholipid antibodies in heart valve disease: a case-control study. Am Heart J 1996;132(4):790-5.

[44] Kampolis C, Tektonidou M, Moyssakis I, et al. Evolution of cardiac dysfunction in patients with antiphospholipid antibodies and/or antiphospholipid syndrome: a 10-year follow-up study. Semin Arthritis Rheum 2014;43(4):558-65.

[45] Stojanovich L, Markovic O, Marisavljevic D, et al. Influence of antiphospholipid antibody levels and type on thrombotic manifestations: results from the Serbian National Cohort Study. Lupus 2012;21(3):4338.

[46] Krause I, Lev S, Fraser A, Blank M, Lorber M, Stojanovich L, et al. Close association between valvar heart disease and central nervous system manifestations in the antiphospholipid syndrome. Ann Rheum Dis 2005;64(10):1490-3.

[47] Espinola-Zavaleta N, Montes RM, Soto ME, et al. Primary antiphospholipid syndrome: a 5-year transesophageal echocardiographic follow-up study. J Rheumatol 2004;31:2402-7.

[48] Amoroso A, Cacciapaglia F, De Castro S, et al. The adjunctive role of antiphospholipid antibodies in systemic lupus erythematosus cardiac involvement. Clin Exp Rheumatol 2006;24:287-94.

[49] Roldan CA, Qualls CR, Sopko KS, et al. Transthoracic versus transesophageal echocardiography for detection of Libman-Sacks endocarditis: a randomized controlled study. J Rheumatol 2008;35:224-9. [50] Mattos P, Santiago MB. Association of antiphospholipid antibodies with valvulopathy in systemic lupus erythematosus: a systematic review. Clin Rheumatol 2011;30(2):165-71. 
[51] Zuily S, Regnault V, Selton-Suty C, et al. Increased risk for heart valve disease associated with antiphospholipid antibodies in patients with SLE. Meta-analysis of echocardiographic studies. Circulation 2011;124:215-22.

[52] Diógenes MJ, Diógenes PC, de Morais Carneiro RM, et al. Cutaneous manifestations associated with antiphospholipid antibodies. Int J Dermatol 2004;43(9):632-7.

[53] Francès C, Niang S, Laffitte E, et al. Dermatologic manifestations of the antiphospholipid syndrome: two hundred consecutive cases. Arthritis Rheum 2005;52(6):1785-93.

[54] Toubi E, Krause I, Fraser A, Lev S, Stojanovich L, Rovensky J, et al. Livedo reticularis is a marker for predicting multi-system thrombosis in antiphospholipid syndrome. Clin Exp Rheumatol 2005;23(4):499-504.

[55] Krause I, Leibovici L, Blank M, et al. Clusters of disease manifestations in patients with antiphospholipid syndrome demonstrated by factor analysis. Lupus 2007; 16(3):176-80.

[56] Gómez-Puerta JA, Martin H, Amigo MC, et al. Long-term follow-up in 128 patients with primary antiphospholipid syndrome: do they develop lupus? Medicine (Baltimore) 2005;84(4):225-30.

[57] García-Carrasco M, Galarza-Maldonado C, Gomez-Ponce M, et al. Antiphospholipid syndrome in Latin American patients: clinical and immunologic characteristics and comparison with European patients. Lupus 2007;16(5):366-7.

[58] Mejía-Romero R, Garcia-Carrasco M, Galarza-Maldonado C, et al. Primary antiphospholipid syndrome in Latin American mestizo patients: clinical and immunologic characteristics and comparison with European patients. Clin Rheumatol 2008;27(7):891-7.

[59] Williams FM, Cherkas LF, Bertolaccini ML, et al. Migraine and antiphospholipid antibodies: no association found in migraine-discordant monozygotic twins. Cephalalgia 2008;28(10):1048-52.

[60] Cavestro C, Micca G, Molinari F, et al. Migraineurs show a high prevalence of antiphospholipid antibodies. J Thromb Haemost 2013;9(7):1350-4.

[61] Asherson RA, Derksen RH, Harris EN, et al. Chorea in systemic lupus erythematosus and "lupuslike" disease: association with antiphospholipid antibodies. Semin Arthritis Rheum 1987;16(4):253-9. [62] Usugi T, Nakano K, Nakayama T, et al. Familial antiphospholipid antibody in a child with involuntary movement and deterioration. Pediatr Int 2007;49(2):238-41.

[63] Orzechowski NM, Wolanskyj AP, Ahlskog JE, Kumar N, Moder KG. Antiphospholipid antibodyassociated chorea. J Rheumatol 2008;35(11):2165-70.

[64] Reiner P, Galanaud D, Leroux G, et al. Long-term outcome of 32 patients with chorea and systemic lupus erythematosus or antiphospholipid antibodies. Mov Disord 2011;26(13):2422-7.

[65] Verrot D, San-Marco M, Dravet C, et al. Prevalence and signification of antinuclear and anticardiolipin antibodies in patients with epilepsy. Am J Med 1997;103(1): 33-7.

[66] Cimaz R, Romeo A, Scarano A, et al. Prevalence of anti-cardiolipin, anti-beta2 glycoprotein I, and anti-prothrombin antibodies in young patients with epilepsy. Epilepsia 2002;43(1):52-9.

[67] Peltola JT, Haapala A, Isojärvi JI, Auvinen A, Palmio J, Latvala K. Antiphospholipid and antinuclear antibodies in patients with epilepsy or new-onset seizure disorders. Am J Med 2000;109(9):712-7.

[68] Liimatainen S, Peltola M, Sabater L, Fallah M, Kharazmi E, Haapala AM, et al. Clinical significance of glutamic acid decarboxylase antibodies in patients with epilepsy. Epilepsia 2010;51(5):760-7. 
[69] Herranz MT, Rivier G, Khamashta MA, Blaser KU, Hughes GR. Association between antiphospholipid antibodies and epilepsy in patients with systemic lupus erythematosus. Arthritis Rheum 1994;37(4):57-568.

[70] Mackworth-Young CG, Hughes GR. Epilepsy: an early symptom of systemic lupus erythematosus. J Neurol Neurosurg Psychiatry 1985;48(2):185.

[71] Toubi E, Khamashta MA, Panarra A, Hughes GR. Association of antiphospholipid antibodies with central nervous system disease in systemic lupus erythematosus. Am J Med 1995;99(4):397-401.

[72] Liou H-H, Wang C-R, Chen C-J, et al. Elevated levels of anticardiolipin antibodies and epilepsy in lupus patients. Lupus 1996;5:307-12.

[73] Kavassa FB, Ioannidis JP, Touloumi G, Boki KA, Moutsopoulos HM. Risk factors for central nervous system involvement in systemic lupus erythematosus. QJM 2000; 93(3):169-74.

[74] Mok CC, Lau CS, Wong RW. Neuropsychiatric manifestations and their clinical associations in southern Chinese patients with systemic lupus erythematosus. J Rheumatol 2001;28:766-71.

[75] Sanna G, Bertolaccini ML, Mathieu A. Central nervous system lupus: a clinical approach to therapy. Lupus 2003;12:935-42.

[76] Appenzeller S, Cendes F, Costallat LTL. Epileptic seizures in systemic lupus erythematosus. Neurology 2004;63:1808-12.

[77] Mikdashi J, Handwerger B. Predictors of neuropsychiatric damage in systemic lupus erythematosus: data from the Maryland lupus cohort. Rheumatology 2004;43: 1555-60.

[78] Sachse C, Lfithke K, Hartung K, et al. Significance of antibodies to cardiolipin in unselected patients with systemic lupus erythematosus, clinical and laboratory associations. Rheumatol Int 1995;15:23-9.

[79] Formiga F, Mitjavila F, Pac M, Moga I. Epilepsy and antiphospholipid antibodies in systemic lupus erythematosus patients. Lupus 1997;6:486.

[80] D'Cruz DP, Mellor-Pita S, Joven B, Sanna G, Allanson J, Taylor J, et al. Transverse myelitis as the first manifestation of systemic lupus erythematosus or lupus-like disease: good functional outcome and relevance of antiphospholipid antibodies. J Rheumatol 2004;31(2):280-5.

[81] Birnbaum JL, Petri M, Thompson R, Izbudak I, Kerr D. Distinct subtypes of myelitis in systemic lupus erythematosus. Arthritis Rheum 2009;60(11):3378-87.

[82] Pengo V, Banzato A, Denas G, Jose SP, Bison E, Hoxha A, et al. Correct laboratory approach to APS diagnosis and monitoring. Autoimmun Rev 2013;12(8):832-4.

[83] Roggenbuck D, Egerer K, von Landenberg P, Hiemann R, Feist E, Burmester GR, et al. Antiphospholipid antibody profiling: time for a new technical approach? Autoimmun Rev 2012;11(11):821-6.

[84] Hughes GRV, Khamashta MA. Seronegative antiphospholipid syndrome. Ann Rheum Dis $2003 ; 62: 1127$.

[85] Jawad ASM. Classification vs diagnostic criteria - a revised international consensus statement on classification criteria for APS is required to accommodate the seronegative clinical entity. Ann Rheum Dis $2004 ; 5: 608$. 
[86] Mahler M, Norman GL, Meroni PL, Khamashta M. Autoantibodies to domain 1 of beta 2 glycoprotein 1: a promising candidate biomarker for risk management in antiphospholipid syndrome. Autoimmun Rev 2012;12(2):313-7.

[87] Meijide H, Sciascia S, Sanna G, Khamashta MA, Bertolaccini ML. The clinical relevance of IgA anticardiolipin and IgA anti- $\beta 2$ glycoprotein I antiphospholipid antibodies: a systematic review. Autoimmun Rev 2013;12(3):421-5.

[88] Bertolaccini ML, Amengual O, Andreoli L, et al. 14th International Congress on Antiphospholipid Antibodies Task Force. Report on antiphospholipid syndrome laboratory diagnostics and trends. Autoimmun Rev 2014;13(8):917-30.

[89] Cervera R, Conti F, Doria A, et al. Does seronegative antiphospholipid syndrome really exist? Autoimmun Rev 2012;11:581-4.

[90] Rodriguez-Garcia JL, Bertolaccini ML, Cuadrado MJ, Sanna G, Ateka-Barrutia O, Khamashta MA. Clinical manifestations of antiphospholipid syndrome (APS) with and without antiphospholipid antibodies (the so-called 'seronegative APS'). Ann Rheum Dis 2012;71(2):242-4.

[91] Petri M. Update on anti-phospholipid antibodies in SLE: the Hopkins' Lupus Cohort. Lupus 2010;19:419-23.

[92] Salcido-Ochoa F, Cabiedes Alarcón-Segovia D, Cabral AR. Antiprothrombin Antibodies in patients with systemic lupus erythematosus or with primary antiphospholipid syndrome. J Clin Rheumatol 2002;8(5):251-5.

[93] Conti F, Alessandri C, Sorice M, et al. Thin-layer chromatography immunostaining in detecting antiphospholipid antibodies in seronegative anti-phospholipid syndrome. Clin Exp Immunol 2012;167(3):429-37.

\section{Table 1.}

Association of seizures in with lupus anticoagulant in SLE: Hopkins Lupus Cohort.

\begin{tabular}{|c|c|c|c|}
\hline \multirow[t]{4}{*}{ Seizures } & LAC positive $(\mathrm{N}=591)$ & LAC negative $(\mathrm{N}=1615)$ & $p$-Value \\
\hline & $13 \%$ & $8.5 \%$ & 0.0017 \\
\hline & aCL positive $(\mathrm{n}=591)$ & aCL negative $(\mathrm{N}=1615)$ & $p$-Value \\
\hline & $9.4 \%$ & $10.2 \%$ & 0.5658 \\
\hline \multirow[t]{4}{*}{ Seizures without stroke } & LA positive $(\mathrm{N}=493)$ & LA negative $(\mathrm{N}=1515)$ & $p$-Value \\
\hline & $9.4 \%$ & $7.7 \%$ & 0.22 \\
\hline & aCL positive $(\mathrm{N}=591)$ & aCL negative $(\mathrm{N}=1615)$ & $p$-Value \\
\hline & $7.4 \%$ & $8.8 \%$ & 0.25 \\
\hline
\end{tabular}


Table 2. Result of the complete critical analysis.

Criteria

Recurrent thrombotic events, including life threatening event

Recurrent thrombotic events, including life threatening event

APS Nephropathy

Valve heart lesions

Livedo reticularis

Neurological

manifestations

Migraine

Chorea

Seizures

Longitudinal myelitis
Kidney failure, death by kidney failure

Heart failure, valve replacement, death by heart failure

Recurrent thrombotic events, including life threatening event

Recurrent thrombotic events, including life threatening event

Stroke, neurological arterial and venous thrombosis

Stroke, neurological arterial and venous thrombosis

Stroke, neurological arterial and venous thrombosis

Recurrent thrombotic events, including life threatening event critical for decision-
Relevance category of PIO

Overall

GRADE

analysis

Important but not

Low making

Critical for decision making

Critical for decision making

Critical for decision making

\section{Important but not} critical for decisionmaking

\section{Important but not} critical for decisionmaking

Critical for decision making

Critical for decision making

Critical for decision making

Critical for decision making

Low

Low

Low

Low
Recommendation $b$ and $c$

Suggestion to be included

Moderate

Moderate

Moderate

Very low
Recommended to be included

Strongly recommended to be included

Strongly recommended to be included

Recommended to be included

Not recommended to be included

Recommended to be included

Not recommended to be included

Recommended to be included

Recommended to be included 